\title{
INTERMEDIATE FREQUENCY ERROR IDENTIFICATION OF OPTICAL ELEMENTS UNDER SVM
}

\author{
Dan Cheng ${ }^{1}$, Zhaohai Yang ${ }^{1}$, Rd Rohmat Saedudin ${ }^{2}$ \\ ${ }^{1}$ Basic teaching and Research Department, Changchun Guanghua University \\ Changchun city Jilin Province 130000, China \\ ${ }^{2}$ School of Industrial Engineering, Telkom University, 40257, Bandung, West Java, Indonesia \\ Email:danchbtrd133@163.com
}

\begin{abstract}
The paper is to improve the identification effect of the intermediate frequency (IF) errors of optical elements. The optical surface frequency band error detection and its characteristics are analysed. The GA-BPANN (Genetic Algorithm- Back Propagation Artificial Neural Network) algorithm and the DAG-SVM (Directed Acyclic Graph- Support Vector Machine) algorithm are proposed in this study. The IF errors of optical elements are also identified separately. Furthermore, the identification accuracy and efficiency of the two algorithms are compared. On both samples based on its length, i.e. small-scale training sample or a large-scale training sample, the DAG-SVM algorithm has a more significant advantage in the identification accuracy rate. In terms of training time, the DAG-SVM algorithm is far lower than the GA-BPANN algorithm. Whereas, in terms of identification time, the GA-BPANN algorithm is significantly lower than the DAG-SVM algorithm. The DAG-SVM algorithm has a higher value in the identification of IF errors of optical elements. This study solves the problem of low identification rate of traditional methods and guides the identification of the IF error in optical element quality detection.
\end{abstract}

Keywords: SVM, Optical Elements, Intermediate Frequency Error Identification, Aspheric Surface, BPANN.

\section{Introduction}

Optical processing manufacturing technology is a long-standing and emerging science. In the past, the processing of optical elements mostly relied on the skills and experience of the processor. Whereas, the accuracy of the finished product was low, and the processing time was long [1]. With the continuous development and optimization of computer science, with computer control as the lead, the method of establishing mathematical models to process optical elements has gradually replaced the traditional optical processing manufacturing technology.

Aspheric optical elements have the advantages of low price, compact structure and excellent optical performance, which have been widely used in optical telescopes [2]. In extreme engineering projects such as large optical telescope systems, surface shape errors will have a significant impact on the performance of optical elements. Among them, the high-frequency surface shape error will cause the light to be scattered at a large angle, and the reflectance of the mirror surface will be reduced. The IF surface shape errors will cause small-angle scattering of light and affect the contrast of the image.
The low-frequency surface shape errors will distort the system's imaging and fetch various aberrations $[3,4]$. Therefore, the problem suppresses the surface shape error of optical elements has become one of the urgent tasks in the research of extreme optical systems.

Computer Controlled Optical Surfacing (CCOS) is an important method for precision and ultraprecision machining of modern optical elements. The entire optical element surface is polished by a set processing track. Because the function of the small grinding head is not continuous, it forms a significant IF error on the surface of the optical element. It also severely affects the energy concentration and imaging quality of the optical system [5-6]. Therefore, it is especially important to control the IF error. Shahinian et al. (2017) explored the feasibility of using optical fiber-based tools to process optical materials and reduce the amplitude of IF surface error in a computer numerical control environment [7]. The recognition of IF errors of optical elements often has low identification accuracy and come up with significant errors. Therefore, Wang et al. (2018) proposed a support vector machine (SVM) algorithm combined with the least square method to be used in hyperspectral imaging systems, which improved the 
accuracy rate of identifying IF errors of optical elements [8]. The SVM algorithm is a machine algorithm based on the VC (Vapnik-Chervonenkis) dimension theory. It can add a regularization term to the solution system to optimize structural risk, and it is a classifier with sparsity and robustness. Also, it has been widely used in pattern identification problems, such as portrait identification and text classification [9-10].

In short, to further improve the identification accuracy of the IF error of the optical element, this paper optimizes and improves the SVM algorithm and applies it to the identification of the IF error of the optical element. The innovation of this paper lies in the improvement of the traditional classical algorithm, and an optimization algorithm is proposed to identify the IF error of the optical element. It solves the problem of low identification rate of traditional methods and provides guidance for the identification of the IF error in optical element quality detection.

\section{Method}

\subsection{Optical surface frequency band error detection method and its characteristics}

This paper uses digital wave surface interferometer to detect data for analysis to study the characteristics of optical surface frequency band errors. First, the discrete frequency band error sampling analysis is performed. According to the Nyquist sampling theorem, to prevent distortion of the continuous signal after sampling, the sampling frequency must be greater than twice the highest frequency of the signal [11-12]. That is, the length of the detection sampling period should be less than or equal to half of the analysis target period, as shown in equation (1).

$$
2 T_{s} \leq T_{o}
$$

Where: $T_{s}$ represents the length of the sampling period. $T_{o}$ represents the analysis target period. When performing aspherical full-caliber detection, it is necessary to meet equation (2).

$$
F \#<R \#
$$

Where: $F \#$ represents the interferometer standard mirror. $R \#$ represents the mirror surface. In the aspheric surface detection, the resolution of the detected mirror surface is inconsistent due to the projection distortion. To ensure the mirror space frequency does not produce a difference error, correction of projection distortion should be performed.

The maximum value in the detection area is taken as the sampling length to reduce the sampling rate. Also, according to the lowest sampling rate, the element surface frequency band error is calculated.
Based on the definition of the projection distortion data point density, the distortion rate can be expressed as the ratio of the minimum data density to the maximum density caused by the distortion. After the digital interferometer samples, the discretized distortion rate is expressed as the ratio of the lengthiest sample to the shortest for interference detection. In addition, the noise has a significant impact on the detection results of band errors. The noise detected by the interferometer is a random disturbance, and the difference can be eliminated by averaging multiple detections. The detection method is as follows: After turning on the interferometer and waiting for about 20 minutes, Zoom is set to the maximum value, and the standard mirror is adjusted to the minimum detection area. Then, the detection of the standard mirror is repeated multiple times within a small interval. The detection results are saved and the detection time is recorded.

Based on the optical surface frequency band error detection, the characteristics of IF errors are analysed. In the evaluation of optical systems, the wavefront slope distribution is an important parameter, and its mean square can reflect the level of the IF error. The main factor affecting the wavefront slope is the vector height slope of the mirror surface. The unidirectional slope of the optically processed surface is defined as equation (3).

$$
\left\{\begin{array}{l}
\mathrm{g}_{x}(x, y)=\frac{\partial S(x, y)}{\partial x} \\
\mathrm{~g}_{y}(x, y)=\frac{\partial S(x, y)}{\partial y}
\end{array}\right.
$$

Where: $S(x, y)$ represents the IF surface shape error. $g_{x}(x, y)$ represents the slope in the $x$ direction. $g_{y}(x, y)$ represents the slope in the $y$ direction.

The wave front slope is expressed as equation (4).

$$
\mathrm{g}_{x y}(x, y)=\sqrt{\mathrm{g}_{x}(x, y)^{2}+\mathrm{g}_{y}(x, y)^{2}}
$$

Where: $g_{x y}(x, y)$ represents the slope of the surface shape in the $x$ and $y$ directions.

The Weierstrass-Mandelbrot (WM) function is used to analyse the fractal characteristics of the surface [13]. The structure function is defined as the mean square error of the surface vector height increment to calculate the fractal dimension, and the discretized structure function is obtained, as shown in equation (5).

$$
D(n \delta)=\frac{1}{N-n} \sum_{m=0}^{N-n}(s(m+n)-s(m))^{2}
$$

Where: $\delta$ represents the increment of a specific direction along the surface. $s(m+n)$ and $s(m)$ represent the vector height of a certain generatrix of the surface contour. $m+n$ and $m$ represent coordinates on the generatrix. 


\subsection{Back Propagation Artificial Neural Network (BPANN) optimized by Genetic Algorithm (GA)}

The core algorithm of BPANN is the extended form of Back Propagation (BP) algorithm. This algorithm adjusts the threshold and connection weight of each node in the network by gradient descent method.

It reduces the error between the actual output value of the network and the expected output value, obtaining the actual output of the network that approximates the expected output [14-15]. Its topology is shown in Figure 1.

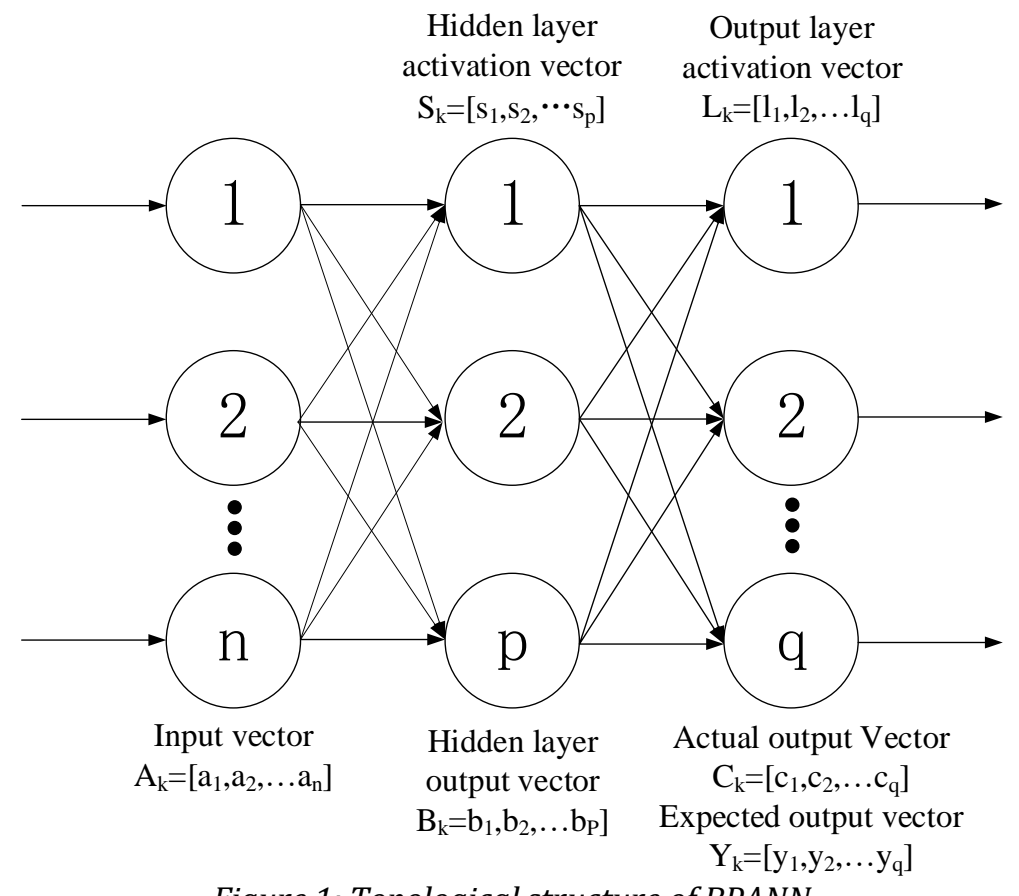

Figure 1: Topological structure of BPANN

First, it is necessary to assign values to each neuron node and its connection weight in the network, as shown in equation (6).

$$
f(x)=\frac{1}{1+e^{-x}}
$$

Where: $x$ represents the input.

The characteristic vector is entered for training, and the activation function of each node in the network is used to obtain the error between the actual output and the expected output. Through the gradient descent method, the threshold and connection weight correction values of the output layer and the middle layer are obtained. Also, the threshold and connection weight of the nodes are adjusted in the network. Then, the characteristic vector is re-entered to determine whether the error is within the specified threshold. If error is not within the threshold limit, the characteristic vector needs to be re-entered for training and adjustment until the network converges appropriate results.

In the BPANN algorithm, the correction value is obtained by the gradient descent method, which results in slow convergence. To improve the network training speed, the adjustment value of the previous weight is multiplied by the adjustment coefficient and accumulated into the current adjustment value, to achieve the purpose of rapid correction. At the same time, on multi-class classification problems, the
BPANN algorithm can easily fall into local minimum points, as shown in Figure 2. Therefore, this paper applies GA algorithm to the selection of initial connection weights and thresholds of BPANN algorithm to form a fusion algorithm, namely GABPANN algorithm. In essence, the GA algorithm is a directed random process that can solve the local extremum problem of the BPANN algorithm.

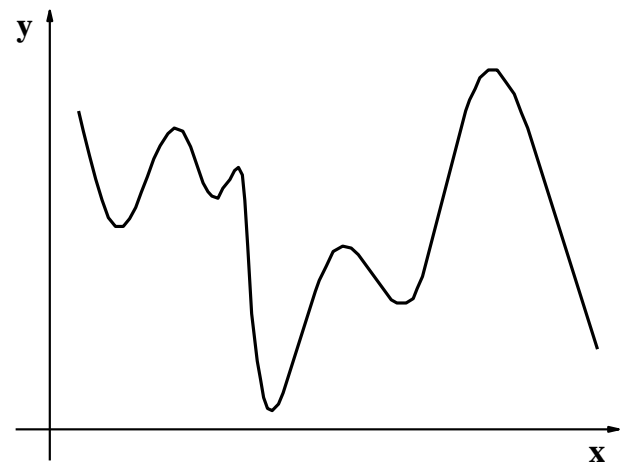

Figure 2: Schematic diagram of BPANN algorithm falling into local minimum points

The GA algorithm first initializes the population with random numbers in the global solution space. Individuals in the population are encoded to obtain encoded strings. 
The fitness function calculates the fitness of individuals in the initial population. Based on the selection rule, it gets depart the bad and remains excellent.

The crossover operations and mutation operations are performed to generate a new generation of candidate populations. The genetic operation is repeated until the convergence index is met, and the algorithm ends.
The GA-BPANN algorithm takes the output error in the BPANN algorithm as the fitness function of the GA algorithm, and uses the GA algorithm to optimize the initial connection weights and thresholds of the BPANN algorithm to obtain the optimal or suboptimal initial value. BPANN is trained, and finally, a suitable network model is obtained. The flowchart of GA-BPANN algorithm is shown in Figure 3.

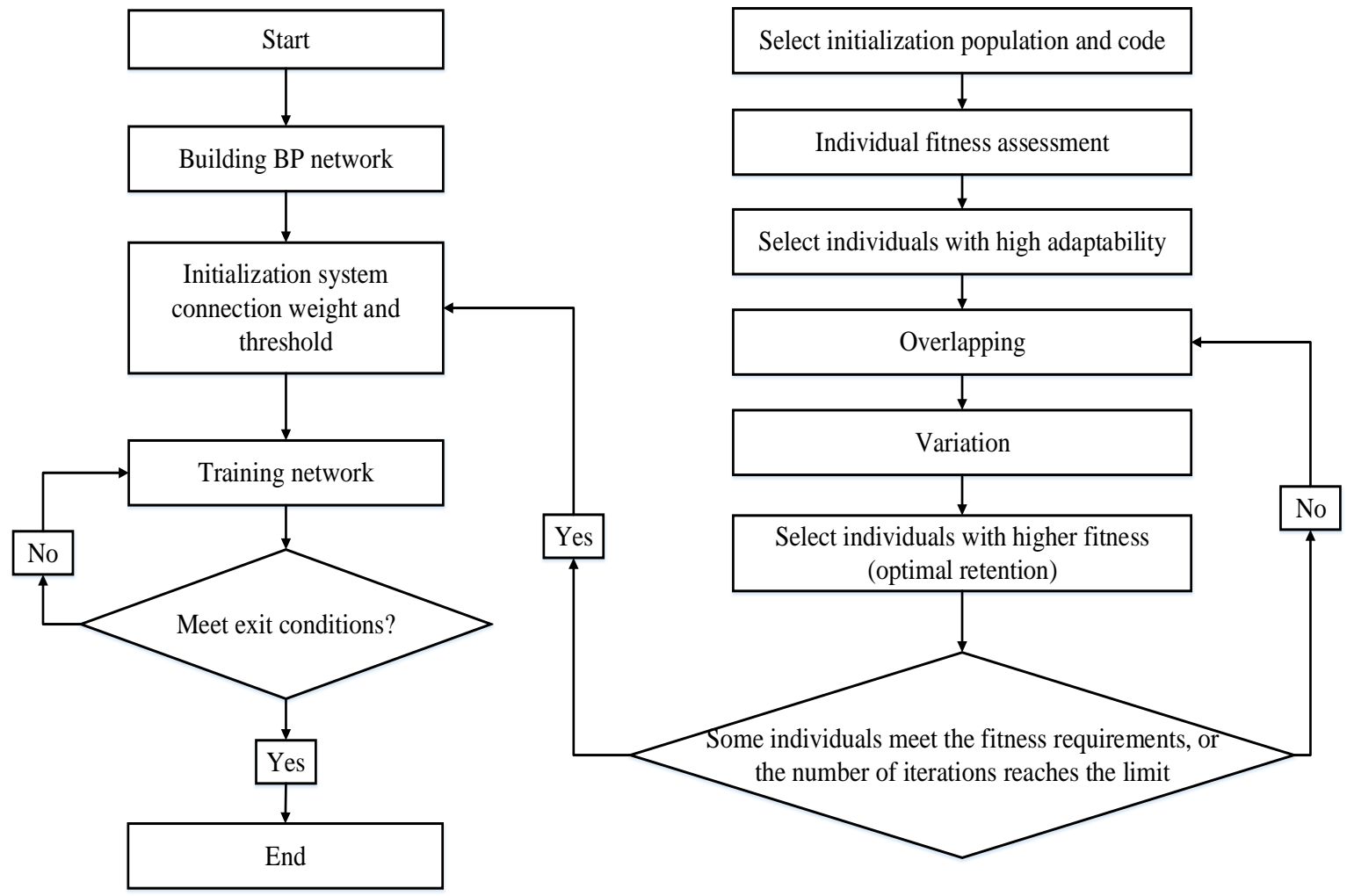

Figure 3: Flowchart of GA-BPANN algorithm

\subsection{SVM algorithm optimized by Directed Acyclic Graph (DAG) method}

The SVM algorithm is an algorithm that transforms a non-linear problem into a problem that a linear perceptron can handle. It is one of the most widely used algorithms in machine learning and suitable for solving the problem of small sample pattern classification [16-17]. In the linear SVM, the training sample set $D$ is defined as equation (7).

$$
D=\left\{\left(x_{1}, y_{1}\right), \mathrm{K},\left(x_{l}, y_{l}\right)\right\}, x \in R^{n}, y \in\{-1,1\}
$$

Taking a two-dimensional training sample as an example, the straight line in Figure 4 represents a linear classifier, and the distance between two dashed lines represents a classification interval. Therefore, a classification surface with an ample interval is a useful classification surface. Equation (8) can be obtained through geometric derivation.

$$
\delta_{\mathrm{i}}=\frac{1}{\|w\|} \bullet\left|g\left(x_{\mathrm{i}}\right)\right|
$$

Where: $\|w\|$ represents the 2-norm of $w .\left|g\left(x_{\mathrm{i}}\right)\right|$ represents the absolute value of $i$ sample on the hyperplane.

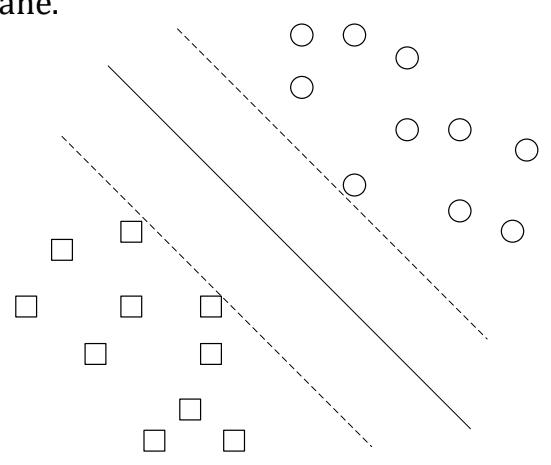

Figure 4: Linear classifier

Since the training sample set cannot be divided between two dashed lines, the problem of optimal classification hyperplane can be transformed into an optimization problem with inequality constraints. 
At the same time, to avoid individual error samples in the training samples from affecting the classification hyperplane solution results, penalty

$$
\begin{aligned}
& \frac{1}{2} \bullet\|\mathrm{w}\|^{2}+C \bullet \sum_{i=1}^{l} \xi_{i} \\
& y_{i} \bullet\left[<w, x_{i}>+b\right]-1+\xi_{i}>=0\left(\xi_{i}>0, i=1,2, \mathrm{~L} l\right)
\end{aligned}
$$

Where: $C$ represents the penalty factors. $\xi_{i}$ represents the relaxation variable. The size of the $C$ value determines the penalties for wrongly divided samples.

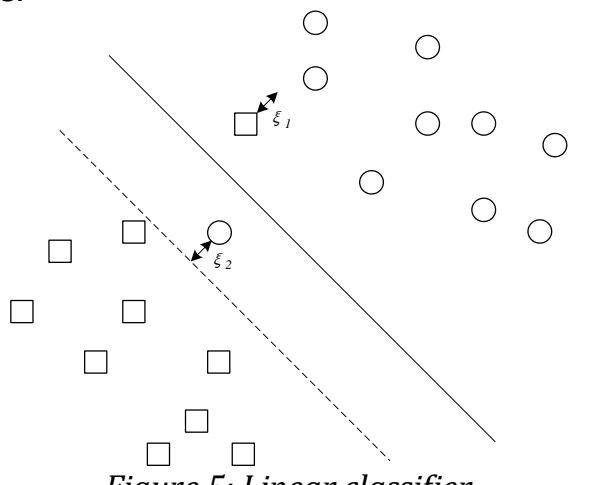

Figure 5: Linear classifier

The decision function is derived as equation (11).

$$
f(x)=\operatorname{sgn}\left(\left(w^{*} \cdot x\right)+b^{*}\right)
$$

Where: $w^{*}$ represents the hyperplane normal vector. $b^{*}$ represents the constant hyperplane translation amount.

In the non-linear SVM, the non-linear problem in the low-dimensional space is transformed into the linearly separable problem in the high-dimensional space by adding a kernel function.
The schematic diagram of the function of the kernel function is shown in Figure 6.

The points between the $\mathrm{AB}$ line are classified as positive, and the points outside the $\mathrm{x}$-axis $\mathrm{AB}$ line are classified as negative. Classification can be performed through a parabola (non-linear classifier). It can convert the original two-dimensional training samples into four-dimensional training samples.

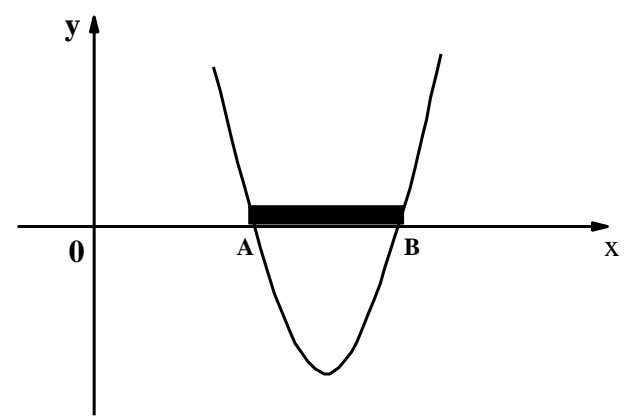

Figure 6: Function diagram of the kernel function

Similarly, the example in the figure is derived from a multi-dimensional case, obtaining equation (12).

$$
\min _{\alpha} \frac{1}{2} \bullet \sum_{i=1}^{l} \sum_{j=1}^{l} y_{i} \cdot y_{j} \cdot \alpha_{i} \cdot \alpha_{j} \cdot K\left(x_{i}, y_{j}\right)-\sum_{j=1}^{l} \alpha_{j}
$$

Where: $y$ represents the expected output of the training samples. $\left(x_{i}, y_{j}\right)$ represents the training sample. $K()$ represents the kernel operation on the input sample vector.

The discriminant function is shown in equation (13).

$$
f(x)=\operatorname{sgn}\left(\sum_{i=1}^{l} \alpha_{i}^{*} \bullet y_{j} \bullet K\left(x_{i}, x\right)+b^{*}\right)
$$

Where: $\alpha^{*}$ represents the Lagrange multiplier, and $b^{*}$ represents the constant hyperplane translation amount.

The value of the function is not qualitatively defined. In this paper, Gaussian function is used as the kernel function of nonlinear support vector machine, as shown in equation (14).

$$
K\left(x, x_{i}\right)=\tanh \left(1-\left(x, x_{i}\right)^{2} / \sigma^{2}\right)
$$

From the above operations, binary classification problems can be obtained. To further solve the multi-class classification problem, this paper introduces the DAG method to the SVM algorithm to form the DAG-SVM algorithm. The DAG-SVM network structure is shown in Figure 7. First, the DAG is topologically sorted to determine a linear order between the nodes. The points can be processed once in the order of the topological sort. Each time a point is processed, the relaxation operations on all edges starting from that point are performed. A test sample only needs to be classified by $\mathrm{k}$ (number of categories for classification problems) binary classifiers to obtain the result. 


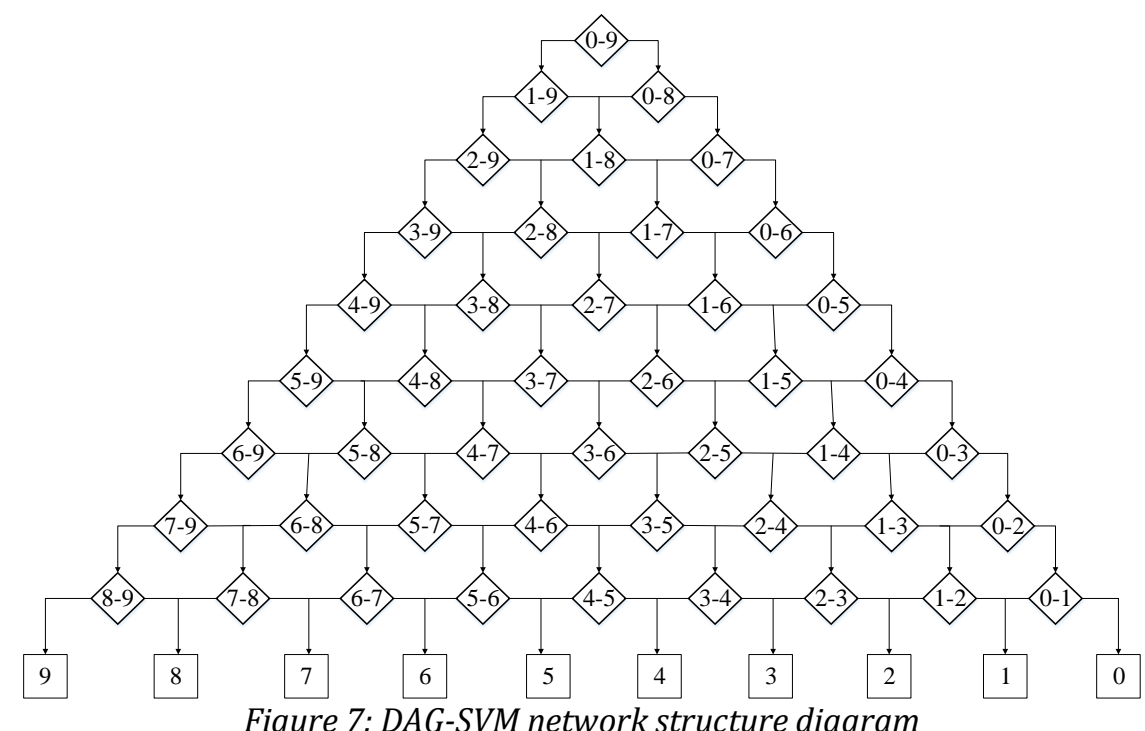

\subsection{Algorithm design}

First, the BPANN algorithm is designed. The three layers of BPANN structure, hidden layer and output layer node activation functions are selected. The steepest gradient descent method is used to adjust the connection weights and thresholds. And the standard back propagation algorithm is used. The value of the learning coefficient is $0.01-0.8$, and the value of the momentum parameter is $0-1$. In actual applications, appropriate adjustments are made according to system performance. The error threshold of a single sample is 0.001 . The error of the entire sample is 50, and the iteration limit is 5000 . Then, the GA-BPANN algorithm is designed to optimize the initial connection weight and node threshold of BPANN. An 8-bit binary code represents the value of each node. The reciprocal of the output error of BPANN after 300 steps of training is used as a fitness function. The individual with the highest fitness is directly inherited to the next generation, and the other individuals perform genetic operations. The 30 individuals with the highest fitness are selected as the population of the next generation among the parent and cross-mutated offspring. Then, it continues to iterate until the fitness meets the requirements of the rule, or the number of iterations reaches the limit, ending the GA algorithm and transferring to the BPANN training part.

The DAG-SVM algorithm is used to identify the IF errors of optical elements. First, the Gaussian kernel function is selected as the kernel function of the nonlinear support vector machine. 5000 samples are selected as training samples to train the classifier, and the identification accuracy rate is required to be higher than 95\%. Also, the obtained penalty coefficient and relaxation variables are used. If the identification accuracy cannot meet the requirements, it increases or decreases by 0.01 steps until the identification accuracy reaches the requirements. DAG is used to build trained binary classifiers. 1000 samples are selected as test samples, and characteristic vectors are extracted from them, testing the identification accuracy of the DAG-SVM algorithm.

\section{Results and Discussion}

The test results of the GA-BPANN algorithm and DAG-SVM algorithm are shown in Table 1 and Figure 8. From Figures $8 \mathrm{~A}$ and $8 \mathrm{~B}$, under a small-scale training sample, the identification accuracy rate of the DAG-SVM algorithm is significantly higher than that of the GA-BPANN algorithm, and the difference between the two is large. Under large-scale training samples, the DAG-SVM algorithm has a higher identification accuracy than the GA-BPANN algorithm, but both have a identification accuracy higher than 90\%. Theoretical analysis shows that both algorithms have strong nonlinear classification ability under large-scale training samples. Under small-scale training samples, the DAG-SVM algorithm starts from the global optimal solution to the convex quadratic programming problem. Thus, the results obtained are better than the global suboptimal solution obtained by the GA-BPANN algorithm. Therefore, whether it is a small-scale training sample or a large-scale training sample, the DAG-SVM algorithm has more enormous advantages.

The comparison of the training time of the two algorithms is shown in Figure 8C. It can be seen that the DAG-SVM algorithm is far lower than the GABPANN algorithm in training time, whether it is a small-scale training sample or a large-scale training sample. It shows that the DAG-SVM algorithm has fewer input characteristics and fast execution speed, which effectively improves the efficiency of IF error identification of optical elements. The comparison of the identification time of the two algorithms is shown in Figure 8D. It can be seen that GA-BPANN algorithm is significantly lower than DAG-SVM 
algorithm in identification time. When the training samples increase, the identification time of the DAGSVM algorithm will increase exponentially. It shows that GA-BPANN algorithm has more advantages in application places with high real-time requirements.
Based on the above analysis, the DAG-SVM algorithm proposed in this paper has obvious advantages in identification accuracy and training time but needs to be further optimized in identification time.

Table 1. Test results of GA-BPANN algorithm and DAG-SVM algorithm

\begin{tabular}{|c|c|c|c|c|c|c|}
\hline Algorithm & \multicolumn{3}{|c|}{ Small-scale training sample } & \multicolumn{2}{c|}{ Large-scale training sample } \\
\cline { 2 - 7 } & $\begin{array}{c}\text { Identification } \\
\text { accuracy rate } \\
(\%)\end{array}$ & $\begin{array}{c}\text { Training } \\
\text { time (min) }\end{array}$ & $\begin{array}{c}\text { Identification } \\
\text { time (ms) }\end{array}$ & $\begin{array}{c}\text { Identification } \\
\text { accuracy rate } \\
(\%)\end{array}$ & $\begin{array}{c}\text { Training } \\
\text { time (min) }\end{array}$ & $\begin{array}{c}\text { Identification } \\
\text { time (ms) }\end{array}$ \\
\hline GA-BPANN & 25.11 & 207 & 22 & 90.64 & 520 & 31 \\
\hline DAG-SVM & 66.43 & 18 & 197 & 94.19 & 49 & 345 \\
\hline
\end{tabular}

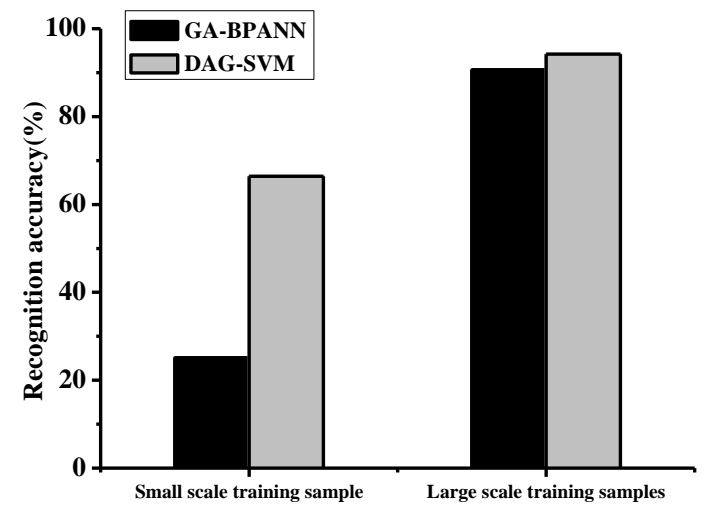

A

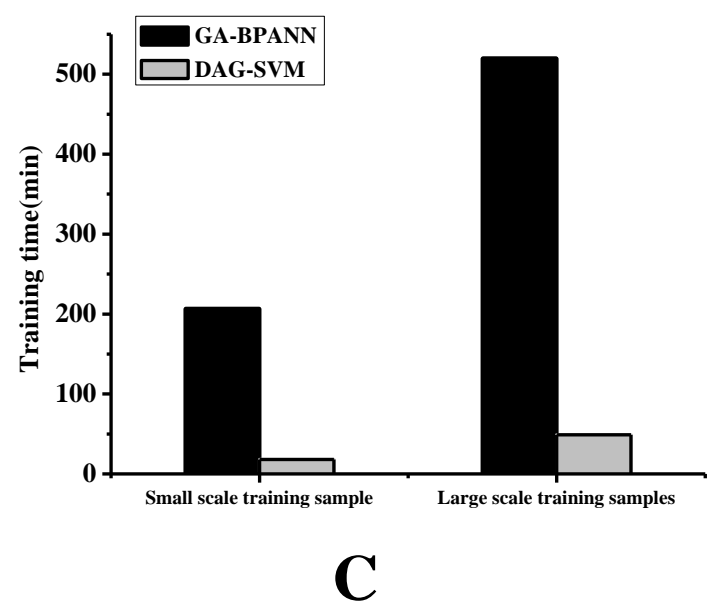

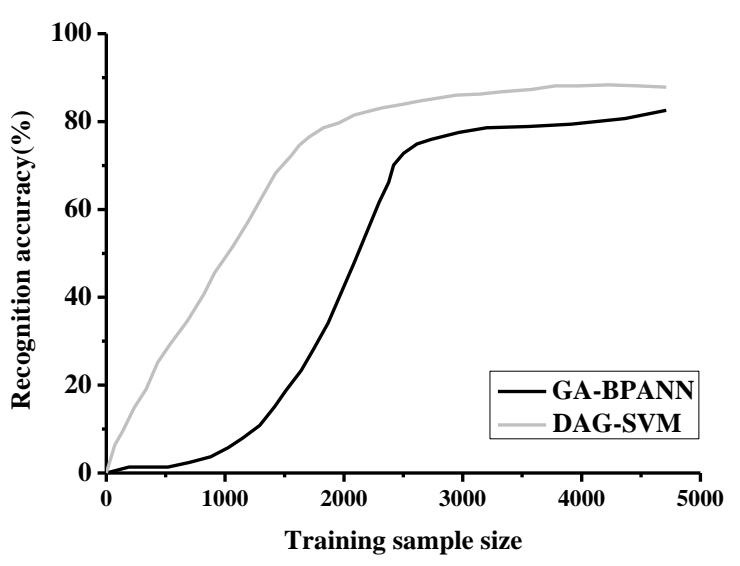

B

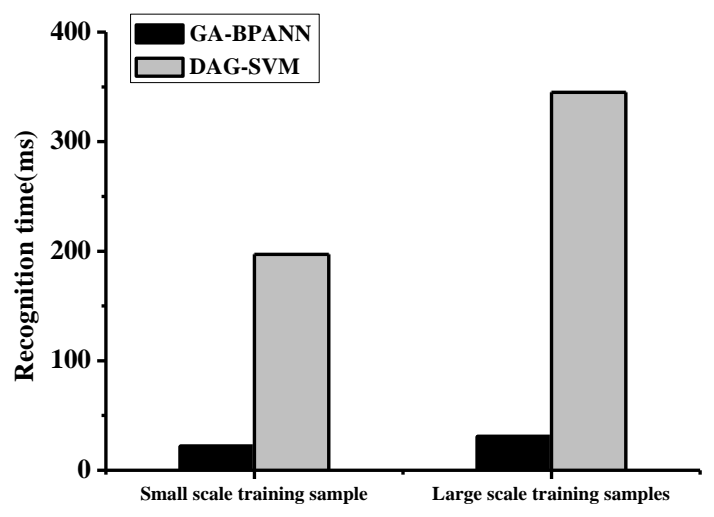

D

Figure 8: Test results of GA-BPANN algorithm and DAG-SVM algorithm (A: Comparison of identification accuracy of the two algorithms; B: Relation diagram of identification accuracy and training sample size; C: Comparison of training time of the two algorithms; D: Two Comparison of identification time of the two algorithms)

\section{Conclusions}

To improve the identification accuracy of the IF error of the optical element, this paper detects the frequency error of the optical surface and analyses its characteristics. Moreover, the GA-BPANN algorithm and DAG-SVM algorithm are proposed. The two algorithms are applied to the IF error identification of optical elements.
Also, the identification accuracy rate, training time and identification time are compared. The results show that the DAG-SVM algorithm has better identification accuracy and training time than the GA-BPANN algorithm, but the identification time of the GA-BPANN algorithm is better than the DAG-SVM algorithm. Therefore, the DAG-SVM algorithm has a high value in the identification of IF errors of optical elements. 
This paper guides for improving the identification accuracy of IF errors in optical elements, but there are some shortcomings in the research process. It only compares the DAG-SVM algorithm with the GABPANN algorithm and does not analyse the accuracy rate of other ANN fusion algorithms in optical element IF error identification. Therefore, in the later research process, it is necessary to apply other ANN fusion algorithms to the IF error identification of optical elements to obtain more reference results.

\section{References}

[1] Ye J, Chen L, Li X, et al. Review of optical freeform surface representation technique and its application[J]. Optical Engineering, 2017, 56(11), pp.110901.

[2] Chen Z, Jiang H Z, Liu X, et al. Measurement of surface defects of optical elements using digital holography[J]. Guangxue Jingmi Gongcheng/Optics and Precision Engineering, 2017, 25(3), pp.576-583.

[3] Tao X, Zhang Z, Zhang F, et al. A novel and effective surface flaw inspection instrument for large-aperture optical elements [J]. IEEE Transactions on Instrumentation and Measurement, 2015, 64(9), pp.2530-2540.

[4] Thiébaut F, Lacroix C, Andolfatto L, et al. Evaluation of the shape deviation of non rigid parts from optical measurements[J]. The International Journal of Advanced Manufacturing Technology, 2017, 88(5-8), pp. 1937-1944.

[5] Nie X, Li S, Hu H, et al. Control of mid-spatial frequency errors considering the pad groove feature in smoothing polishing process[J]. Applied Optics, 2014, 53(28), pp. 6332.

[6] S.K. Gugulothu, N. Prabhu Kishore, V. Phani Babu, Girish Sapre (2019). Cfd Analysis on Different Piston Bowl Geometries by Using Split Injection Techniques. Acta Mechanica Malaysia, 2(1): 2328.

[7] Shahinian H, Hassan M, Cherukuri H, et al. Fiberbased tools: material removal and mid-spatial frequency error reduction[J]. Applied Optics, 2017, 56(29), pp.8266.
[8] Wang W, Huang M, Zhu Q, et al. Optical property inversion of biological materials using Fourier series expansion and LS-SVM for hyperspectral imaging[J]. Inverse Problems in Science and Engineering, 2018, 26(7), pp.1019-1036.

[9] Kadhum Audaa Jehhef and Mohamed Abed Al Abas Siba (2019). Effect Of Surfactant Addition On The Nanofluids Properties: A Review. Acta Mechanica Malaysia, 2(2): 1-19, DOI: 10.26480/amm.02.2019.01.19

[10] Cao J, Yuan Y, Su L, et al. Adaptive Bad Pixel Correction Method for Interference-Modulated Images Based on Weighted Least Squares Support Vector Machines (WLS-SVM)[J]. Applied spectroscopy, 2019, 73(4), pp.454-463.

[11] Syed Mamun R Rasid, Md. Belayet Hossain, Md. Emdadul Hoque, Md. Ariful Azam Arif And Md. Sarikat Ali Sarder (2019). Modeling and Control of a Magnetic Levitation System Using an Analog Controller. Acta Electronica Malaysia, 3(2): 41-44.

[12] Madiena C, Faurie J, Porée J, et al. Color and vector flow imaging in parallel ultrasound with sub-Nyquist sampling[J]. IEEE transactions on ultrasonics, ferroelectrics, and frequency control, 2018, 65(5), pp.795-802.

[13] Dong J, Ju Y, Gao F, et al. Estimation of the fractal dimension of Weierstrass-Mandelbrot function based on Cuckoo search methods[J]. Fractals, 2017, 25(06): 1750065.

[14] Ahmed M. Alturas, Abdulmajed O. Elbkosh, Othman Imrayed (2020). Stability Analysis of DC-DC Buck Converters. Acta Electronica Malaysia, 4(1): 01-06.

[15] Hughes T W, Minkov M, Shi Y, et al. Training of photonic neural networks through in situ backpropagation and gradient measurement[J]. Optica, 2018, 5(7): 864-871.

[16] Cheng J, Tao D, Wong D W K, et al. Quadratic divergence regularized SVM for optic disc segmentation[J]. Biomedical optics express, 2017, 8(5): 2687-2696.

[17] Akbar Zada, Hafiz Ullah (2019). Decomposition Of $\mathrm{Cm}$ Through Q-Periodic Discrete Evolution Family. Matrix Science Mathematic, 3(1): 09-12. 